\title{
Unexpected intramolecular cyclization of 4-(2-halophenyl)-1H- pyrazolo[3,4-b]quinolines: formation of 5- and 7-membered rings from one starter
}

\author{
Krzysztof S. Danel, ${ }^{\mathrm{a},}$ *Anna Wisła, ${ }^{a}$ and Tomasz Uchacz ${ }^{\mathrm{b}}$ \\ ${ }^{a}$ Department of Chemistry, University of Agriculture, Balicka St. 122, 30-149 Kraków, Poland \\ ${ }^{b}$ Faculty of Chemistry, Jagiellonian University, Ingardena St. 3, 30-060 Kraków, Poland \\ E-mail: rrdanelk@cyf-kr.edu.pl
}

\begin{abstract}
Cyclization of 4-(2-halophenyl)-1H-pyrazolo[3,4-b]quinolines 3a-b and 9a-9b provided two regioisomeric compounds: 6-phenyl-6H-5,6,7-triazadibenzo[f,h]naphtho[3,2,1-cd]azulenes 4,10 and 1,3-diphenyl-3H-indeno[1,2,3-de]pyrazolo[3,4-b]quinolines 2,11. All of them are considered as new building blocks for optoelectronic materials. The two-step synthesis utilized readily available starting materials.
\end{abstract}

Keywords: Azafluoranthene, annulation, cyclodehydrohalogenation, 1H-pyrazolo[3,4b]quinoline

\section{Introduction}

Pyrazolo[3,4-b]quinolines (PQs) are known for their antiviral (HSV-1, HSV-2, CMV, VZ and EB) properties, ${ }^{1}$ as COX inhibitors, ${ }^{2}$ inhibitors of oncogenic Ras, ${ }^{3}$ interferon inducing agents, ${ }^{4}$ antimicrobials, ${ }^{5}$ antimalarials, ${ }^{6}$ and potent apoptosis inducers. ${ }^{7}$ PQs also belong to a class of highly fluorescent compounds which emit mostly in the blue spectral range and have been classified as promising materials for optoelectronics. ${ }^{8}$ Attempts have been made to red-shift of their fluorescence spectra, ${ }^{9}$ by changing substituents and introduction of the additional N-atom into the central aromatic ring. In this project we have paid attention to polycyclic aromatic hydrocarbons (PAHs) of a high photoluminescence quantum yields useful for OLEDs. ${ }^{10}$ Generally speaking, an increase in the extent of the $\pi$-electron system (i.e. degree of conjugation) leads to a shift of the absorption and fluorescence spectra to longer wavelengths and to an increase in the fluorescence quantum yield. ${ }^{11}$ In the case of PQs the only possibility left to achieve the expected effect is to increase the number of fused rings. In this paper we disclose a practical synthesis of azafluoranthenes and heterocyclic analogues of azulene derived from PQs. 
These new heterocycles could be potential building blocks in supramolecular chemistry and luminophores for OLEDs. ${ }^{12}$

\section{Results and Discussion}

Since prerequisite PQ 1 could not be simply prepared by the method formerly developed, ${ }^{13}$ which eventually would allow the formation of 2, its isomeric 4-(2-chlorophenyl)-1,3-diphenyl-1Hpyrazolo[3,4-b]quinoline 3a was prepared from 2-chlorobenzaldehyde, 1,3-diphenylpirazol-5one and aniline. The isomer was then cyclized according to Clar. ${ }^{14}$ Instead of the expected derivative of 3-azafluoranthene $\mathbf{2}$, only a seven-membered heterocycle $\mathbf{4}$ was formed. To confirm our result, we prepared 4-(2-chlorophenyl)-3,7-dimethyl-1-phenyl-1H-pyrazolo[3,4-b]quinoline 5. The attempted cyclodehydrohalogenation in $\mathbf{5}$ on reflux in quinoline/KOH failed. In another approach to facilitate the ring closure we also synthesized 7, but its cyclization did not yield any traces of 8 (Scheme 1).
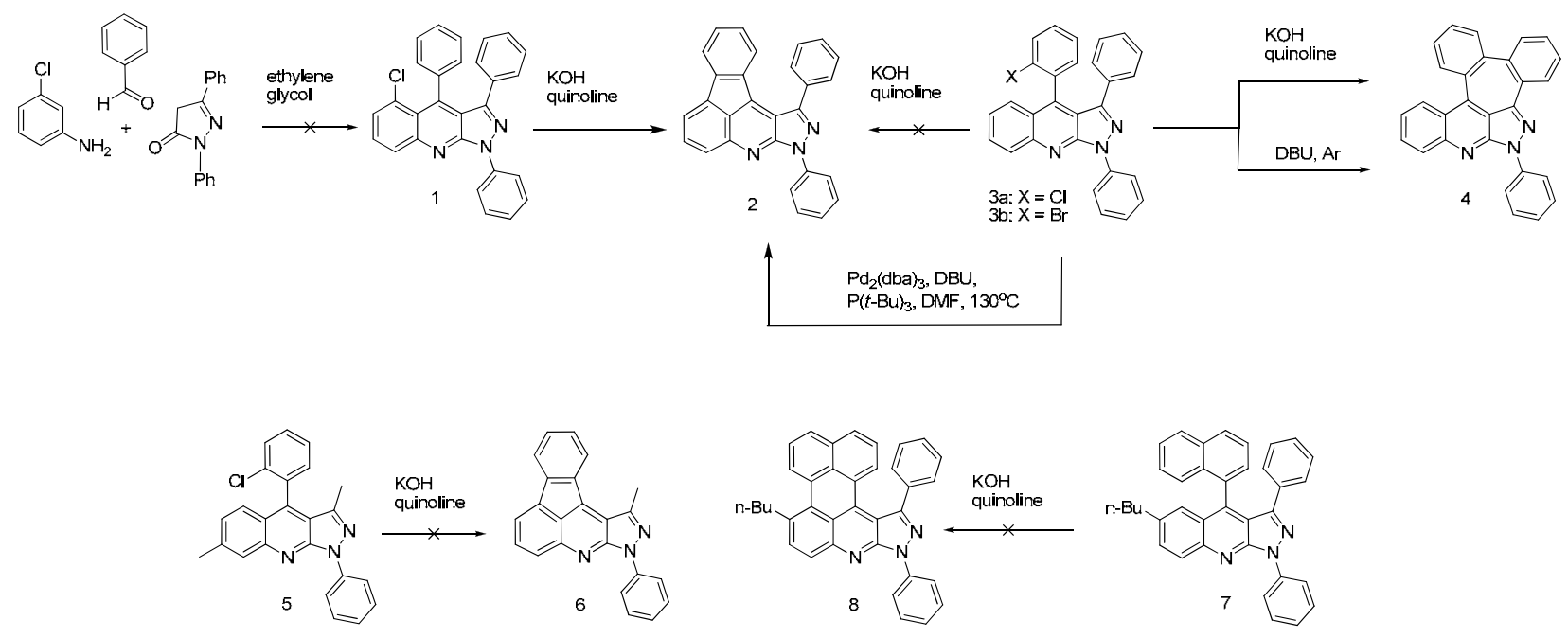

\section{Scheme 1}

Prolonged reflux of $\mathbf{3 a , b}$ in quinoline did not produce nor $\mathbf{2}$ or $\mathbf{4}$. Therefore, the presence of halogen atom and a strong inorganic base appeared indispensable for the ring closure. Our attempt involving the use of a powerful hindered organic base, 1,8-diazabicyclo[5.4.0]undec-7ene (DBU) in different solvents, ${ }^{15}$ as suggested in the literature did not give appreciable results because of the uncompleted conversion to 4 . Finally, we found that the easiest approach to the synthesis of the regioisomer 2 involved cyclization of 4-(2-bromophenyl)-1,3-diphenyl-1Hpyrazolo[3,4-b]quinoline $\mathbf{3 b}$ by modified Wegner $^{16}$ procedure where $\mathrm{P}(t-\mathrm{Bu})_{3}$ was used as a ligand instead of $\mathrm{P}(\mathrm{Cy})_{3}$. This reaction furnished indeno-annelated product 2 with a reasonable yield and only a trace of seven-membered ring system $\mathbf{4}$ was formed (TLC monitoring). Contrary 
to it, heating of $\mathbf{3 b}$ in $\mathrm{KOH} /$ quinoline gave exclusively $\mathbf{4}$. The same reactions conditions were also examined for 3a. This delivered a mixture of $\mathbf{2}$ and $\mathbf{4}$, with a low yield. More recently, the group of Scott has proven that even the chlorine atom could be utilized in the intramolecular cyclization using different Pd catalysts. ${ }^{17}$ To additionally confirm the site of cyclization, we readily obtained two halo derivatives $\mathbf{9 a , b}$ (Scheme 2 ). They were treated in the same manner as mentioned above. The characterization of $\mathbf{1 0}$ and $\mathbf{1 1}$ was achieved by NMR spectroscopy according to the following strategy: i) the spectrum of the regioisomer $\mathbf{1 1}$ revealed two doublets

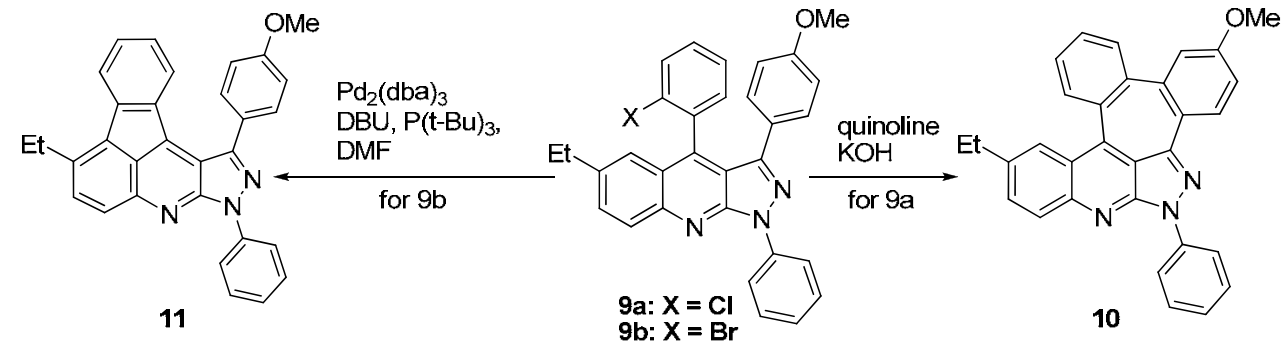

\section{Scheme 2}

originating from a free 3-p-methoxyphenyl moiety; ii) the analysis of $\mathbf{1 0}$ provided evidence for the cross-annulation of the 3-ring. The plausible explanation accounting for the formation of 4 (or 10) is depicted below (Scheme 3): 3a,b (9a,b) treated with $\mathrm{KOH}$ gives a reactive intermediate (o-benzyne) 13a. In the present case 13a has the possibility of reacting in two different orientations: to close the five-membered ring or to attack the adjacent phenyl moiety. The latter route seems to be more probable to bring the aryne within bonding distance. Thus a diradical 13b attacks the 3-phenyl ring creating $\sigma$ bond with hydrogen abstraction.

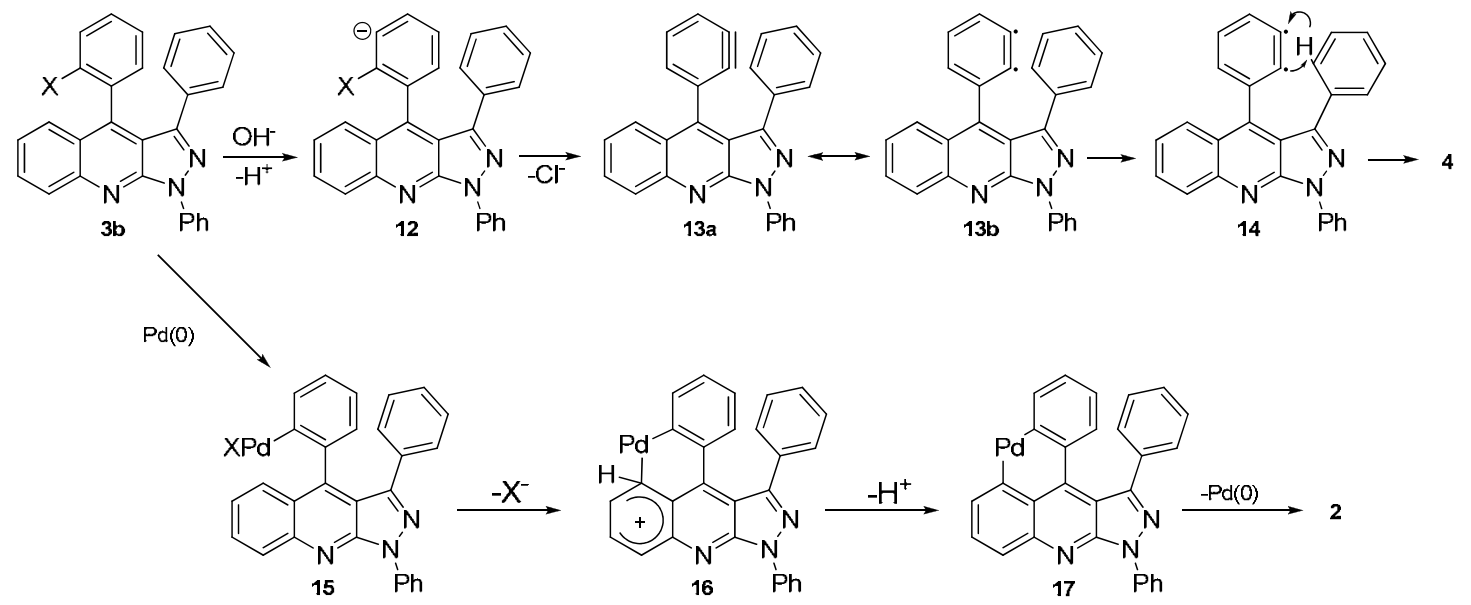

\section{Scheme 3}

Similar transformation was observed by Cram and coworkers. ${ }^{18}$ Indeno[1,2,3]-annelated 2 (or 11) products are examples of an intramolecular arylation to form the five-membered rings. It is 
often considered that such cyclization involves intramolecular electrophilic attack of the arylpalladium(II) moiety $\mathbf{1 5}$ on another aromatic ring in the key intermediate. ${ }^{15 b}$ In this case the six-membered geometry of the intermediate $\mathbf{1 6}$ should facilitate this ring closure and disfavor the formation of seven-membered analogues. On the other hand, the presence of the electronwithdrawing $\mathrm{N}$-atom should make this assumption inconsistent with an electrophilic aromaticsubstitution mechanism. Recently, another authors proposed a quite different mechanism proceeding by abstraction of a proton of the arylated ring by the base in a process in which the formation of the metal-carbon bond is concerted with the breaking of the carbon-hydrogen bond. ${ }^{19}$ They studied the intramolecular palladium-catalyzed arylation of on a variety of bromobenzyldiarylmethane systems. They found that electron-withdrawing substituents favored this reaction. In our case the 5-position of $\mathbf{3 b}$ is more acidic because of the electronegative $\mathrm{N}$ atom of the pyridine than ortho-protons of the proximal phenyl ring at the 3-position. As a result this leads to the nearly exclusive formation of five-membered regioisomers.

\section{Conclusions}

In summary, this preliminary report describes two new, efficient synthetic routes to a variety of aromatic structures containing five- and seven-membered rings. The starting materials are cheap, readily available and the compounds can be prepared in 2 steps in most cases without chromatographic separation. The remarkable is the easy formation of seven-membered aromatic ring system which is very rare. ${ }^{20}$ The latter also can be considered as the example of topological chemical defects spotted in carbon nanotubes (pentagon-heptagon pair). ${ }^{21}$ Both annulated structures can be derived from the same starter. Further studies are under way to expand the scope of the present method, and to use the products for further conversion as well as for the OLED construction.

\section{Experimental Section}

\section{1,3-Di(aryl)- and 1-aryl-3-methyl-4-(2-halophenyl)-1H-pyrazolo[3,4-b]quinolines 3. General procedure}

Equimolar amounts $(10 \mathrm{mmol})$ of aniline, 1,3-diphenylpyrazolin-5-one and 2-halobenzaldehyde were refluxed for $2 \mathrm{~h}$ with TLC monitoring in ethylene glycol $(20 \mathrm{~mL})$. After cooling the mixture was treated with $\mathrm{MeOH}$. It was refluxed for $30 \mathrm{~min}$, followed by cooling and sonication in a cold water bath for $1 \mathrm{~h}$. The yellow precipitate was collected. Only bromoderivative $\mathbf{3 b}$ was further purified by column chromatography (alumina, toluene) because it was contaminated with a little amount of debrominated product.

4-(2-Chlorophenyl)-1,3-diphenyl-1H-pyrazolo[3,4-b]quinoline (3a). Light yellow solid; yield $1.64 \mathrm{~g}(38 \%) ; \mathrm{mp}=200-202{ }^{\circ} \mathrm{C} ; \mathrm{UV}(\mathrm{MeCN}): \lambda_{\max }[\mathrm{nm}]=398 \mathrm{~nm}$; Fluorescence $(\mathrm{MeCN}) \lambda_{\max }$ 
$[\mathrm{nm}]=481 ;{ }^{1} \mathrm{H}$ NMR $\left(300 \mathrm{MHz}, \mathrm{CDCl}_{3}\right) \delta$ 7.06-7.25 (m, 7H), 7.26-7.35 (m, 3H), 7.40 (ddd, $1 \mathrm{H}, J=8.7,6.6,1.2 \mathrm{~Hz}), 7.58(\mathrm{t}, 2 \mathrm{H}, J=7.5 \mathrm{~Hz}), 7.64(\mathrm{ddd}, 1 \mathrm{H}, J=8.7,1.5,0.6 \mathrm{~Hz}), 7.78$ (ddd, $1 \mathrm{H}, J=8.7,6.6,1.5 \mathrm{~Hz}), 8.27$ (ddd, $1 \mathrm{H}, J=8.7,1.2,0.6 \mathrm{~Hz}), 8.62(\mathrm{~d}, 2 \mathrm{H}, J=9.0 \mathrm{~Hz}$ ); Anal. Calcd. for $\mathrm{C}_{28} \mathrm{H}_{18} \mathrm{ClN}_{3} ; \mathrm{C}, 77.86 ; \mathrm{H}, 4.20 ; \mathrm{N}$, 9.73. Found: C, 77.39; H, 4.56; N, 9.71.

4-(2-Bromophenyl)-1,3-diphenyl-1H-pyrazolo[3,4-b]quinoline (3b). Light yellow solid; yield $1.67 \mathrm{~g}(35 \%) ; \mathrm{mp}=204-206{ }^{\circ} \mathrm{C} ;{ }^{1} \mathrm{H}$ NMR $\left(300 \mathrm{MHz}, \mathrm{CDCl}_{3}\right) \delta 7.06-7.26(\mathrm{~m}, 8 \mathrm{H}), 7.32(\mathrm{t}, 1 \mathrm{H}$, $J=7.5 \mathrm{~Hz}$ ), 7.39 (ddd, 1H, $J=8.1,6.6,1.5 \mathrm{~Hz}), 7.50-7.53(\mathrm{~m}, 1 \mathrm{H}), 7.57(\mathrm{t}, 2 \mathrm{H}, J=7.5 \mathrm{~Hz})$, $7.62(\mathrm{ddd}, 1 \mathrm{H}, J=9.0,1.2,0.9 \mathrm{~Hz}), 7.77$ (ddd, $1 \mathrm{H}, J=9.0,6.6,1.5 \mathrm{~Hz}), 8.26$ (ddd, $1 \mathrm{H}, J=9.0$, 1.2, $0.9 \mathrm{~Hz}), 8.63\left(\mathrm{~d}, 2 \mathrm{H}, J=8.7 \mathrm{~Hz}\right.$ ); Anal. Calcd. for $\mathrm{C}_{28} \mathrm{H}_{18} \mathrm{BrN}_{3} ; \mathrm{C}, 70.60 ; \mathrm{H}, 3.81 ; \mathrm{N}, 8.82$. Found: C, 70.60; H, 4.17; N, 8.97.

4-(2-Chlorophenyl)-3,7-dimethyl-1-phenyl-1H-pyrazolo[3,4-b]quinoline (5). This compound was prepared following general procedure for compounds 3 using equimolar (10 mmol) of 3methylaniline, 1-phenyl-3-methylpyrazolin-5-one and 2-chlorobenzaldehyde. Yellow solid; yield $1.19 \mathrm{~g} \mathrm{(31 \% );} \mathrm{mp} \mathrm{=} \mathrm{147-148}{ }^{\circ} \mathrm{C} ;{ }^{1} \mathrm{H}$ NMR (300 MHz, $\left.\mathrm{CDCl}_{3}\right) \delta 2.13$ (s, 3H), 2.57 (s, 3H), 7.20 $(\mathrm{dd}, 1 \mathrm{H}, J=8.7,1.8 \mathrm{~Hz}), 7.26(\mathrm{t}, 1 \mathrm{H}, J=7.5 \mathrm{~Hz}), 7.37(\mathrm{dd}, 1 \mathrm{H}, J=7.5,1.8 \mathrm{~Hz}), 7.42-7.57$ (m, $5 \mathrm{H}), 7.62(\mathrm{dd}, 1 \mathrm{H}, J=7.8,1.2 \mathrm{~Hz}), 8.00(\mathrm{t}, 1 \mathrm{H}, J=0.6 \mathrm{~Hz}), 8.51$ (d, 2H, $J=8.7 \mathrm{~Hz})$; Anal. Calcd. for $\mathrm{C}_{24} \mathrm{H}_{18} \mathrm{ClN}_{3} ; \mathrm{C}, 75.09 ; \mathrm{H}, 4.73 ; \mathrm{N}, 10.95$. Found: C, 74.98; H, 4.95; N, 10.93 .

6-Butyl-4-(naphthalen-1-yl)-1,3-diphenyl-1H-pyrazolo[3,4-b]quinoline (7). This compound was prepared following general procedure for compounds 3 using equimolar (10 mmol) of 4-nbutylaniline, 1,3-diphenylpyrazolin-5-one and 1-naphthaldehyde. Light yellow solid; yield $2.45 \mathrm{~g}$ (49\%); mp $=152-153{ }^{\circ} \mathrm{C} ;{ }^{1} \mathrm{H}$ NMR $\left(300 \mathrm{MHz}, \mathrm{CDCl}_{3}\right) \delta 0.80(\mathrm{t}, 3 \mathrm{H}, J=7.2 \mathrm{~Hz}), 1.16-1.28(\mathrm{~m}$, $2 \mathrm{H}), 1.44-1.54(\mathrm{~m}, 2 \mathrm{H}), 2.56(\mathrm{t}, 2 \mathrm{H}, J=7.5 \mathrm{~Hz}), 6.74(\mathrm{t}, 2 \mathrm{H}, J=7.5 \mathrm{~Hz}), 6.80-6.84(\mathrm{~m}, 2 \mathrm{H})$, $6.95(\mathrm{t}, 1 \mathrm{H}, J=7.2 \mathrm{~Hz}), 7.23-7.34(\mathrm{~m}, 6 \mathrm{H}), 7.39-44(\mathrm{~m}, 1 \mathrm{H}), 7.58(\mathrm{t}, 2 \mathrm{H}, J=7.5 \mathrm{~Hz}), 7.64(\mathrm{dd}$, $1 \mathrm{H}, J=9.0,1.8 \mathrm{~Hz}), 7.79-7.83(\mathrm{~m}, 2 \mathrm{H}), 8.21(\mathrm{~d}, 1 \mathrm{H}, J=8.7 \mathrm{~Hz}), 8.64(\mathrm{~d}, 2 \mathrm{H}, J=8.7 \mathrm{~Hz})$; Anal. Calcd. for $\mathrm{C}_{36} \mathrm{H}_{29} \mathrm{~N}_{3} ; \mathrm{C}, 85.85 ; \mathrm{H}, 5.80 ; \mathrm{N}, 8.34$. Found: C, 85.86; H, 5.89; N, 8.55.

\section{6-Ethyl-4-(2-halophenyl)-3-(4-methoxyphenyl)-1-phenyl-1H-pyrazolo[3,4-b]quinoline 9a,b.}

\section{General procedure}

Equimolar amounts $(10 \mathrm{mmol})$ of 4-ethylaniline, 3-(4-methoxyphenyl)-1-phenylpyrazolin-5-one and 2-halobenzaldehyde were refluxed for $2 \mathrm{~h}$ with TLC monitoring in ethylene glycol (20 mL). The mixture was cooled and treated with $\mathrm{MeOH}$. Next it was refluxed for $30 \mathrm{~min}$, followed by cooling and sonication in a cold water bath for $1 \mathrm{~h}$. The yellow precipitate was collected.

\section{4-(2-Chlorophenyl)-6-ethyl-3-(4-methoxyphenyl)-1-phenyl-1H-pyrazolo[3,4-b]quinoline}

(9a). Light yellow solid; yield $2.45 \mathrm{~g}(50 \%) ; \mathrm{mp}=149-151{ }^{\circ} \mathrm{C} ;{ }^{1} \mathrm{H}$ NMR $\left(300 \mathrm{MHz}, \mathrm{CDCl}_{3}\right) \delta$ $1.24(\mathrm{t}, 3 \mathrm{H}, J=7.5 \mathrm{~Hz}), 2.73(\mathrm{q}, 2 \mathrm{H}, J=7.5 \mathrm{~Hz}), 3.77\left(\mathrm{~s}, 3 \mathrm{H}, \mathrm{OCH}_{3}\right), 6.62(\mathrm{~d}, 2 \mathrm{H}, J=8.7 \mathrm{~Hz})$, 7.13-7.20 (m, 4H), 7.27-7.38 (m, 4H), 7.57 (t, 2H, $J=7.5 \mathrm{~Hz}), 7.66(\mathrm{dd}, 1 \mathrm{H}, J=8.7,1.8 \mathrm{~Hz})$, $8.18(\mathrm{~d}, 1 \mathrm{H}, J=9.0 \mathrm{~Hz}), 8.61(\mathrm{~d}, 2 \mathrm{H}, J=8.7 \mathrm{~Hz})$; Anal. Calcd. for $\mathrm{C}_{31} \mathrm{H}_{24} \mathrm{ClN}_{3} \mathrm{O}$; C, 75.99; $\mathrm{H}$, $4.94 ; \mathrm{N}, 8.58$. Found: C, 75.63; H, 5.40; N, 8.55.

4-(2-Bromophenyl)-6-ethyl-3-(4-methoxyphenyl)-1-phenyl-1H-pyrazolo[3,4-b]quinoline (9b). Light yellow solid; yield $2.40 \mathrm{~g}(45 \%) ; \mathrm{mp}=164-165{ }^{\circ} \mathrm{C} ;{ }^{1} \mathrm{H}$ NMR $\left(300 \mathrm{MHz}, \mathrm{CDCl}_{3}\right) \delta$ 
$1.24(\mathrm{t}, 3 \mathrm{H}, J=7.5 \mathrm{~Hz}), 2.73(\mathrm{q}, 2 \mathrm{H}, J=7.5 \mathrm{~Hz}), 3.76\left(\mathrm{~s}, 3 \mathrm{H}, \mathrm{OCH}_{3}\right), 6.62(\mathrm{~d}, 2 \mathrm{H}, J=9.0 \mathrm{~Hz})$,

$7.11-7.24(\mathrm{~m}, 5 \mathrm{H}), 7.30(\mathrm{t}, 1 \mathrm{H}, J=7.5 \mathrm{~Hz}), 7.34(\mathrm{dd}, 1 \mathrm{H}, J=8.1,0.6 \mathrm{~Hz}), 7.54-7.59(\mathrm{~m}, 3 \mathrm{H})$, $7.65(\mathrm{dd}, 1 \mathrm{H}, J=9.0,2.1 \mathrm{~Hz}), 8.18(\mathrm{~d}, 1 \mathrm{H}, J=8.7 \mathrm{~Hz}), 8.62(\mathrm{~d}, 2 \mathrm{H}, J=8.7 \mathrm{~Hz})$; Anal. Calcd. for $\mathrm{C}_{31} \mathrm{H}_{24} \mathrm{BrN}_{3} \mathrm{O} ; \mathrm{C}, 69.67 ; \mathrm{H}, 4.53 ; \mathrm{N}, 7.86$. Found: C, 69.14; H, 4.68; N, 8.07.

\section{Cyclization of 3a and 9a. General procedure}

3a $(0.50 \mathrm{~g}, 1.16 \mathrm{mmol})$, powdered $\mathrm{KOH}(1.95 \mathrm{~g}, 34.8 \mathrm{mmol})$ and quinoline $(20 \mathrm{~mL})$ were refluxed for 3-4 h until reaction was completed (TLC, toluene). After cooling, the orange solution was treated with $10 \%$ hydrochloric acid $(100 \mathrm{~mL})$ and extracted with toluene $(2 \times 20$ $\mathrm{mL}$ ). The organic phase was washed with saturated aqueous $\mathrm{NaCl}$ solution, dried over anhydrous $\mathrm{MgSO}_{4}$ and evaporated to dryness. The residue was dissolved in chloroform and $\mathrm{MeOH}$ was added dropwise on sonication in a cold water bath (1 hour). A precipitate was filtered off.

6-Phenyl-6H-5,6,7-triazadibenzo[f,h]naphtho[3,2,1-cd]azulene (4). Yellow solid; yield 307 mg (67\%); mp = 203-205 ${ }^{\circ} \mathrm{C}$; IR (KBr) 3100, 1597, 1499, 1461, 1400, 1138, 737, $689 \mathrm{~cm}^{-1}$; UV $(\mathrm{MeCN}): \lambda_{\max }[\mathrm{nm}]=446 \mathrm{~nm}$; Fluorescence $(\mathrm{MeCN}) \lambda_{\max }[\mathrm{nm}]=542 ;{ }^{1} \mathrm{H} \mathrm{NMR}(300 \mathrm{MHz}$, $\left.\mathrm{CDCl}_{3}\right) \delta 7.30(\mathrm{t}, 1 \mathrm{H}, J=7.5 \mathrm{~Hz}), 7.38-7.53(\mathrm{~m}, 5 \mathrm{H}), 7.58(\mathrm{t}, 2 \mathrm{H}, J=7.5 \mathrm{~Hz}), 7.70-7.75(\mathrm{~m}$, 2H), 7.80-7.83 (m, 1H), $7.86(\mathrm{dd}, 1 \mathrm{H}, J=8.1,1.2 \mathrm{~Hz}), 8.16(\mathrm{dd}, 1 \mathrm{H}, J=8.4,0.6 \mathrm{~Hz}), 8.31-8.34$ $(\mathrm{m}, 1 \mathrm{H}), 8.44(\mathrm{dd}, 1 \mathrm{H}, J=8.7,0.9 \mathrm{~Hz}), 8.60(\mathrm{~d}, 2 \mathrm{H}, J=8.7 \mathrm{~Hz}) ;{ }^{13} \mathrm{C} \mathrm{NMR}\left(75 \mathrm{MHz}, \mathrm{CDCl}_{3}\right) \delta$ $120.31,120.95,121.82,123.69,125.16,127.45,127.54,127.61,128.62,128.88,129.01,129.11$, 129.52, 129.98, 132.08, 133.47, 134.25, 134.64, 135.40, 138.17, 140.13, 140.68, 142.12, 143.70 , 150.49, 150.65; MS (FAB) $\mathrm{m} / \mathrm{z} 396\left(\mathrm{M}^{+}+\mathrm{H}\right)$; Anal. Calcd. for $\mathrm{C}_{28} \mathrm{H}_{17} \mathrm{~N}_{3}$; C, 85.04; H, 4.33; N,10.63. Found: C, 84.92; H, 4.46; N, 10.61 .

10-Ethyl-2-methoxy-6-phenyl-6H-5,6,7-triazadibenzo[f,h]naphtho[3,2,1-cd]azulene (10). Yellow-orange solid; yield $998 \mathrm{mg}(71 \%) ; \mathrm{mp}=137-139{ }^{\circ} \mathrm{C} ;{ }^{1} \mathrm{H}$ NMR $\left(300 \mathrm{MHz}, \mathrm{CDCl}_{3}\right) \delta$ $1.32(\mathrm{t}, 3 \mathrm{H}, J=7.5 \mathrm{~Hz}), 2.83(\mathrm{q}, 2 \mathrm{H}, J=7.5 \mathrm{~Hz}), 3.91\left(\mathrm{~s}, 3 \mathrm{H}, \mathrm{OCH}_{3}\right), 7.03(\mathrm{dd}, 1 \mathrm{H}, J=9.0,2.7$ $\mathrm{Hz}), 7.27(\mathrm{t}, 1 \mathrm{H}, J=7.5 \mathrm{~Hz}), 7.32(\mathrm{~d}, 1 \mathrm{H}, J=2.7 \mathrm{~Hz}), 7.41(\mathrm{td}, 1 \mathrm{H}, J=7.8,1.5 \mathrm{~Hz}), 7.48(\mathrm{td}$, $1 \mathrm{H}, J=7.5,1.5 \mathrm{~Hz}), 7.55(\mathrm{t}, 2 \mathrm{H}, J=7.5 \mathrm{~Hz}), 7.59(\mathrm{dd}, 1 \mathrm{H}, J=9.0,2.1 \mathrm{~Hz}), 7.76(\mathrm{dd}, 1 \mathrm{H}, J=$ 7.8, $1.5 \mathrm{~Hz}), 7.89(\mathrm{dd}, 1 \mathrm{H}, J=7.8,1.5 \mathrm{~Hz}), 8.08(\mathrm{~d}, 1 \mathrm{H}, J=8.7 \mathrm{~Hz}), 8.22(\mathrm{~d}, 1 \mathrm{H}, J=0.9 \mathrm{~Hz})$, $8.23(\mathrm{~d}, 1 \mathrm{H}, J=8.7 \mathrm{~Hz}), 8.58(\mathrm{~d}, 2 \mathrm{H}, J=8.7 \mathrm{~Hz}) ;{ }^{13} \mathrm{C} \mathrm{NMR}\left(75 \mathrm{MHz}, \mathrm{CDCl}_{3}\right) \delta 15.36,28.98$, $55.46,114.71,116.99$, 120.09, 120.91, 121.82, 124.82, 124.85, 126.81, 127.64, 128.97, 129.19, $129.43,131.38,134.05,134.93,135.20,139.47,139.68,140.32,140.50,141.22,143.64,149.42$, 150.50, 159.89; Anal. Calcd. for $\mathrm{C}_{31} \mathrm{H}_{23} \mathrm{~N}_{3} \mathrm{O}$; C, 82.10; H, 5.11; N, 9.26. Found: C, 82.00; H, $5.49 ;$ N, 9.24 .

\section{Cyclization of $3 \mathrm{~b}$ and $9 \mathrm{~b}$. General procedure}

3b (476 mg, $1 \mathrm{mmol}), \mathrm{Pd}_{2}(\mathrm{dba})_{3}(25 \mathrm{mg}, 0.024 \mathrm{mmol}, 2.4 \% \mathrm{~mol})$ and DBU $(0.30 \mathrm{~mL})$ were placed in a $25 \mathrm{~mL}$ round-bottomed flask. The flask was capped with a rubber septum and dry DMF (15 mL) was added through a syringe. The mixture was flushed with argon for 30 min and $\mathrm{P}(t-\mathrm{Bu})_{3}$ in toluene $(19.4 \mathrm{mg}, 0.096 \mathrm{mmol}, 0.12 \mathrm{~mL})$ was added in one portion. The mixture was stirred for another $10 \mathrm{~min}$ at room temperature then the yellow solution was $3 \mathrm{~h}$ stirred in an oil 
bath at $130^{\circ} \mathrm{C}$ until starting material disappeared. After cooling, the deep brown solution was poured into water $(25 \mathrm{~mL})$. The mixture was extracted with toluene $(2 \times 25 \mathrm{~mL})$. The organic phase was washed with saturated aqueous $\mathrm{NaCl}$ solution and dried over anhydrous $\mathrm{MgSO}_{4}$. After evaporation, the residue was treated with chloroform $(5 \mathrm{~mL})$ and $\mathrm{MeOH}$ was added dropwise on sonication in a cold water bath. The precipitate was filtered off.

1,3-Diphenyl-3H-indeno[1,2,3-de]pyrazolo[3,4-b]quinoline (2). Orange crystals; yield $285 \mathrm{mg}$ $(72 \%) ; \mathrm{mp}=212-214{ }^{\circ} \mathrm{C}$; IR (KBr) 3111, 1599, 1499, 1447, 1403, 1387, 753, $689 \mathrm{~cm}^{-1}$; UV $(\mathrm{MeCN}): \lambda_{\max }[\mathrm{nm}]=437 \mathrm{~nm}$; Fluorescence $(\mathrm{MeCN}) \lambda_{\max }[\mathrm{nm}]=550 ;{ }^{1} \mathrm{H} \mathrm{NMR}(300 \mathrm{MHz}$, $\left.\mathrm{CDCl}_{3}\right) \delta 6.90(\mathrm{dt}, 1 \mathrm{H}, J=7.5,0.6 \mathrm{~Hz}), 7.03(\mathrm{td}, 1 \mathrm{H}, J=7.5,1.2 \mathrm{~Hz}), 7.31(\mathrm{dd}, 1 \mathrm{H}, J=7.5,1.2$ $\mathrm{Hz}), 7.34(\mathrm{t}, 1 \mathrm{H}, J=7.2 \mathrm{~Hz}), 7.55-7.63(\mathrm{~m}, 5 \mathrm{H}), 7.72(\mathrm{dd}, 2 \mathrm{H}, J=4.8,1.2 \mathrm{~Hz}), 7.75-7.83(\mathrm{~m}$, 3H), 7.95-8.00 (m, 1H), $8.53(\mathrm{~d}, 2 \mathrm{H}, J=8.7 \mathrm{~Hz}) ;{ }^{13} \mathrm{C} \mathrm{NMR}\left(75 \mathrm{MHz}, \mathrm{CDCl}_{3}\right) \delta 112.81,118.92$, $121.61,121.65,123.98,125.82,127.72,127.93,128.27,128.54,129.01,129.37,129.90,130.22$, 132.30, 133.90, 137.35, 138.34, 139.82, 139.87, 140.98, 144.79, 145.90, 153.07; MS (FAB) m/z $396\left(\mathrm{M}^{+}+\mathrm{H}\right)$; Anal. Calcd. for $\mathrm{C}_{28} \mathrm{H}_{17} \mathrm{~N}_{3}$ : C, 85.04; H, 4.33; N,10.63; found: C, 85.11; H, 4.37; N, 10.48 .

7-Ethyl-1-(4-methoxyphenyl)-3-phenyl-3H-indeno[1,2,3-de]pyrazolo[3,4-b]quinoline (11). Light yellow crystals; yield $170 \mathrm{mg}(75 \%) ; \mathrm{mp}=200-203{ }^{\circ} \mathrm{C} ;{ }^{1} \mathrm{H}$ NMR $\left(300 \mathrm{MHz}, \mathrm{CDCl}_{3}\right) \delta$ $1.45(\mathrm{t}, 3 \mathrm{H}, J=7.5 \mathrm{~Hz}), 3.21(\mathrm{q}, 2 \mathrm{H}, J=7.5 \mathrm{~Hz}), 3.96\left(\mathrm{~s}, 3 \mathrm{H}, \mathrm{OCH}_{3}\right), 7.08(\mathrm{dd}, 1 \mathrm{H}, J=1.5,0.9$ $\mathrm{Hz}), 7.10(\mathrm{~d}, 1 \mathrm{H}, J=0.6 \mathrm{~Hz}), 7.13(\mathrm{~d}, 2 \mathrm{H}, J=8.7 \mathrm{~Hz}), 7.30-7.42(\mathrm{~m}, 2 \mathrm{H}), 7.57$ (t, 2H, $J=7.5$ $\mathrm{Hz}), 7.60(\mathrm{~d}, 1 \mathrm{H}, J=8.7 \mathrm{~Hz}), 7.73(\mathrm{~d}, 2 \mathrm{H}, J=8.7 \mathrm{~Hz}), 7.90(\mathrm{~d}, 1 \mathrm{H}, J=7.8 \mathrm{~Hz}), 7.96(\mathrm{~d}, 1 \mathrm{H}, J=$ $9.0 \mathrm{~Hz}), 8.54(\mathrm{~d}, 2 \mathrm{H}, J=8.7 \mathrm{~Hz}) ;{ }^{13} \mathrm{C} \mathrm{NMR}\left(75 \mathrm{MHz}, \mathrm{CDCl}_{3}\right) \delta ; 14.84,26.74,55.50,112.50$, $113.97,121.51,123.73,124.18,125.59,126.52$, 126.93, 128.03, 128.51, 128.97, 129.78, 131.50, 133.85, 135.17, 137.49, 138.82, 139.59, 139.99, 141.06, 143.73, 145.57, 152.69, 160.56; Anal. Calcd. for $\mathrm{C}_{31} \mathrm{H}_{23} \mathrm{~N}_{3} \mathrm{O}$; C, 82.10; H, 5.11; N, 9.26. Found: C, 82.00; H, 5.27; N, 9.34.

\section{Acknowledgements}

Dr. Marek Kasprowicz (Dept. of Physics, University of Agriculture) is acknowledged for recording IR spectra.

\section{References}

1. Afonso, A.; Kelly, J. M.; Chackalamannil, S. U.S. Patent 5,506,236, 1996; Chem. Abstr. 1996, 125, $33629 \mathrm{q}$.

2. Uchikawa, O.; Mitsui, K.; Asakawa, A.; Morimoto, S.; Yamamoto, M.; Kimura, H.; Moriya, T.; Mizuno, M. U.S. Patent 6,949,648, 2005; Chem. Abstr. 2005, 143, 306450.

3. Wolin, R.; Wang, D.; Kelly, J.; Afonso, A.; James, L.; Kirschmeier, P.; McPhail, A. T. Bioorg. Med. Chem. Lett. 1996, 6, 195. 
4. Crenshaw, R. R.; Luke, G. M.; Siminoff, P. J. Med.Chem. 1976, 19, 262.

5. El-Sayed, O. A.; Aboul-Enein, H. Y. Arch. Pharm. Pharm. Med. Chem. 2001, 334, 117.

6. Stein, R. G.; Biel, J. H.; Singh, T. J. Med.Chem. 1970, 13, 153.

7. Zhang, H. Z.; Claassen, G.; Crogran-Grundy, C.; Tseng, B.; Drewe, J.; Cai, S. X. Bioorg. Med. Chem. 2008, 16, 222.

8. (a) Danel, A.; He, Z.; Milburn, G. H. W.; Tomasik, P. J. Mater. Chem. 1999, 9, 339. (b) Tao, Y. T.; Balasubramaniam, E.; Danel, A.; Wisla, A.; Tomasik, P. J. Mater. Chem. 2001, 11, 768. (c) FuksJanczarek, I.; Gondek, E.; Kityk, I. V.; Danel, K.; Krzemińska, L.; Sanetra, J.; Kwiecień, B. Spectrochim. Acta, Part A 2006, 63, 320.

9. (a) Tao, J. T.; Balasubramaniam, E.; Danel, A.; Jarosz, B.; Tomasik, P. Appl. Phys. Lett. 2000, 77, 1575. (b) Gondek, E.; Kityk, I. V.; Danel, A.; Wisla, A.; Pokladko, M.; Sanetra, J.; Sahraoui, B. Mat. Lett. 2006, 60, 3301.

10. (a) Mi, B. X.; Gao, Z. Q.; Lee, C. S.; Kwong, H. L.; Wang, N. B.; Lee, S. T. J. Mater. Chem. 2001, 11, 2244. (b) Mi, B. X.; Gao, Z. Q.; Liu, M. W.; Chan, K. Y.; Kwong, H. L.; Wong, N. B.; Lee, C. S.; Hung, L. S.; Lee, S. T. J. Mater. Chem. 2002, 12, 1307.

11. Valeur, B. Molecular Fluorescence: Principles and Applications; Wiley: New York, 2001; pp 54-65.

12. (a) De Backer, S.; Prinzie, Y.; Verheijen, W.; Smet, M.; Desmedt, K.; Dehaen, W.; De Schryver, F.

C. J. Phys. Chem. A 1998, 102, 5451. (b) Smet, M.; Dehaen, W. Molecules 2000, 5, 620. (c) Chen, J.P. U.S. Patent 20040101711, 2004; Chem. Abstr. 2004, 141, 14229.

13. (a) Chaczatrian, K.; Chaczatrian, G.; Danel, A.; Tomasik, P. ARKIVOC 2001, (vi), 63. (b) Danel, A.; Chaczatrian, K.; Tomasik, P. ARKIVOC 2000, (i), 51.

14. (a) Clar, E.; Kelly, W.; Wright, J. W. J. Chem. Soc. 1954, 1108. (b) Clar, E.; Willicks, W. J. Chem. Soc. 1958, 942. (c) Smet, M.; Shukla, R.; Fülöp, L.; Dehaen, W. Eur. J. Org. Chem. 1998, 12, 2769.

15. (a) Wang, L.; Shevlin, P. B. Org. Lett. 2000, 2, 3703. (b) Echavarren, A. M.; Gómez-Lor, B.; González, J. J.; de Frutos, Ó. Synlett 2003, 5, 585. (c) Cheng, X. H.; Höger, S.; Fenske, D. Org. Lett. 2003, 5, 2587.

16. Wegner, H. A.; Scott, L. T.; de Meijere, A. J. Org. Chem. 2003, 68, 883.

17. Jackson, E. A.; Steinberg, B. D.; Bancu, M.; Wakamiya, A.; Scott, L. T. J. Am. Chem. Soc. 2007, 129, 484.

18. (a) Warmuth, R. Chem. Commun. 1998, 1, 59. (b) Beno, B. R.; Sheu, C.; Houk, K. N.; Warmuth, R.; Cram, D. J. Chem. Commun. 1998, 3, 301.

19. Pascual, S.; de Mendoza, P.; Echavarren, A. M. Org. Biol. Chem. 2007, 5, 2727.

20. Kaoudi, T.; Quiclet-Sire, B.; Seguin, S.; Zard, S. Z. Angew. Chem. Int. Ed. 2000, 39, 731.

21. (a) Crespi, V. H.; Cohen, M. L.; Louie, S. G. S.; Zettl, A. K. U. S. Patent 6,835,952, 2004; Chem. Abstr. 2003, 138, 264103. (b) Nasibulin, A. G.; Queipo, P.; Shandakov, S. D.; Brown, D. P.; Jiang, H.; Pikhitsa, P. V.; Tolochko, O. V.; Kauppinen, E. I. J. Nanosci. Nanotechnol. 2006, 6, 1233. (c) Ponomareva, I.; Chernozatonskii, L. A.; Andriotis, A. N.; Menon, M. N. J. Phys. 2003, 5, 119.1. (d) Bronstein, H. E.; Scott. L. T. J. Org. Chem. 2008, 73, 88. 